\title{
EDUCAÇÃO PERMANENTE E QUALIFICAÇÃO PROFISSIONAL PARA ATENÇÃO BÁSICA
}

\section{Kênia Souto Moreira}

Enfermeira, Mestre em Cuidado Primário em Saúde, Universidade Estadual de Montes Claros, Brasil.

E-mail: cassioenf2014@gmail.com

\section{Cássio de Almeida Lima}

Enfermeiro, Mestrando pelo Programa de Pós-graduação em Saúde, Sociedade e Ambiente (PPGSaSa), Faculdade de Ciências Biológicas e da Saúde, Universidade Federal dos Vales do Jequitinhonha e Mucuri, Diamantina (MG), Brasil.

\section{Maria Aparecida Vieira}

Enfermeira, Doutora em Ciências; Docente, Programa de Pós-graduação em Cuidado Primário em Saúde, Departamento de Enfermagem, Universidade Estadual de Montes Claros (MG), Brasil.

\section{Simone de Melo Costa}

Cirurgiã-Dentista, Doutora em Odontologia-Saúde Coletiva. Docente, Programa de Pós-graduação em Cuidado Primário em Saúde, Departamento de Odontologia, Universidade Estadual de Montes Claros, (MG), Brasil.
RESUMO: Objetivou-se analisar a avaliação das equipes de saúde da família quanto aos itens educação permanente e qualificação profissional para Atenção Básica. Pesquisa quantitativa, transversal e analítica, realizada em município polo ao norte de Minas Gerais. Os dados de 75 equipes foram coletados a partir da autoavaliação para melhoria do acesso e da qualidade da atenção básica, em 2014. Consideraramse dois itens de padrão de qualidade em educação permanente e qualificação. A análise estatística foi realizada no Software IBM SPSS 22.0. Processaram-se análises bivariadas considerando o nível de significância $p<0,05$. Predominou a autoavaliação positiva, com média de 15,2 pontos, sendo $53,3 \%$ das equipes classificadas no padrão muito satisfatório, $26,7 \%$ satisfatório e $20,0 \%$ regular, com melhor classificação para as equipes da zona urbana comparadas às rurais $(p<0,05)$. A educação permanente ainda não se efetivou no cotidiano dos profissionais. Sugere-se incentivar a qualificação profissional para aquisição de competências e habilidades na atenção básica.

PALAVRAS-CHAVE: Desenvolvimento de pessoal; Avaliação de serviços de saúde; Qualidade da assistência à saúde; Atenção primária à saúde.

\section{PERMANENT EDUCATION AND PROFESSIONAL QUALIFICATION FOR BASIC HEALTH CARE}

\begin{abstract}
Family health teams were evaluated with regard to permanent education and professional qualification for basic health care. Current quantitative, transversal and analytic research was undertaken in a municipality in the north of the state of Minas Gerais, Brazil, where data on 75 teams were collected in 2014 through the SelfEvaluation for the Improvement of Access and Quality of Basic Care. Two items for quality standard in permanent education and qualification were taken into account. Statistical analysis was performed by IBM SPSS 22.0, with bi-variate analyses at $p<0.05$ significance level. Positive selfevaluation predominated, with mean 15.2 marks, or rather, $53.3 \%$ for teams classified as very satisfactory; $26.7 \%$ as satisfactory; $20.0 \%$ as fair, with higher classification for the urban teams rather than the rural ones $(p<0.05)$. Permanent education is still an item on the agenda of professionals. Professional qualification is encouraged for the acquisition of competence and capacity in Basic Health Care.
\end{abstract}

KEY WORDS: Personal development; Assessment of health service; Quality of assistance in health; Basic health care. 


\section{INTRODUÇão}

A avaliação deve ser parte do processo de trabalho em saúde, a fim de propiciar mudanças em suas dimensões de produtividade, satisfação e realização pessoal dos profissionais e dos usuários, economia de meios e otimização de recursos, de modo a adequálos à realidade na qual estão inseridos (YAMAMOTO; MACHADO; SILVA JUNIOR, 2015). Nesse processo de avaliação encontra-se, atualmente, o Programa Nacional de Melhoria do Acesso e da Qualidade da Atenção Básica (PMAQ), em que a equipe de saúde é autora e alvo de avaliação do próprio trabalho, em um processo dialógico e de monitoramento de indicadores para a apropriação da realidade do território. Torna-se possível, assim, o diagnóstico das necessidades da população atendida; a identificação dos nós críticos de acesso e o incentivo para o aprimoramento na produção das informações assistenciais, com aperfeiçoamento do processo de trabalho das equipes, culminando na melhoria da qualidade da atenção (BRASIL, 2013; PORTELA et al., 2013).

O PMAQ tem na educação permanente em saúde uma de suas principais apostas, tanto para a produção de movimentos locais quanto na singularização, suporte, qualificação e reinvenção no movimento de mudança das práticas de atenção, gestão, educação e participação (PINTO; SOUSA; FLORÊNCIO, 2012; AURÉLIO PINTO et al., 2014). A educação permanente em saúde insere-se em uma necessária constituição de relações e processos que partem do interior das equipes em atuação conjunta às práticas organizacionais, conforme Ceccim (2005).

Para Seidl et al. (2014), a oferta de educação permanente aos profissionais de saúde se constitui em uma ferramenta potente de gestão do trabalho, o que contribui não apenas para a valorização e satisfação do trabalhador, como também para melhor qualificação do processo de trabalho. É nesse contexto em que se insere a Política Nacional de Educação Permanente em Saúde, proposta de prática pedagógica que coloca o cotidiano do trabalho em saúde como central aos processos educativos, ao mesmo tempo em que o coloca sob problematização, em autoanálise e autogestão (BRASIL, 2004; YAMAMOTO; MACHADO; SILVA JUNIOR, 2015).
Ademais, a educação permanente se ancora na interface aprendizagem e trabalho, por meio do desenvolvimento profissional e institucional, da educação para o conhecimento e educação para o trabalho, construção do saber e invenção de práticas no trabalho (AURÉLIO PINTO et al., 2014).

O esforço de análise do PMAQ sobre as questões de educação permanente em saúde na Atenção Básica (AB) pode ser uma contribuição para a busca de maior potencial de transformação das práticas de saúde. Estudos devem ser utilizados para aprofundar essa questão, além de evidenciar informações sobre ofertas mais singulares de práticas de educação permanente e a avaliação que os profissionais fazem delas (AURÉLIO PINTO et al., 2014; CARDOSO et al., 2015). As investigações sobre o assunto têm contribuído para seu aprimoramento, ao fornecer subsídios para reflexões que irão possibilitar mudanças nas práticas de atenção à saúde (LEITE et al., 2012).

Este estudo teve como objetivo analisar a avaliação das equipes de saúde da família quanto aos itens educação permanente e qualificação profissional para $\mathrm{AB}$.

\section{METODOLOGIA}

Trata-se de estudo com abordagem quantitativa, desenho transversal e analítico, realizado em município polo ao norte do Estado de Minas Gerais, de porte médio, e é a quinta maior cidade desse Estado, com contingente populacional estimado em 394.350 mil habitantes (IBGE, 2015).

Os dados de todas as 75 equipes de saúde da família, cadastradas no município em 2014, foram coletados a partir da Autoavaliação para Melhoria do Acesso e da Qualidade da Atenção Básica (AMAQ), em site do Ministério da Saúde, após impressos disponibilizados por técnicos da Secretaria Municipal de Saúde para fins do estudo.

A AMAQ é um instrumento referente à segunda fase de desenvolvimento do PMAQ. É uma ferramenta de avaliação de serviços de saúde gerada a partir de instrumentos validados, nacionalmente e internacionalmente, e utilizada pelo Ministério da Saúde 
brasileiro. O instrumento é composto por duas dimensões relacionadas à equipe de $\mathrm{AB}$, com análise independente. A primeira dimensão trata da Unidade Básica de Saúde (UBS) e a segunda da educação permanente, processo de trabalho e atenção integral à saúde. Essa última dimensão contém a subdimensão considerada neste estudo, educação permanente e qualificação das equipes de AB. São avaliados dois padrões de qualidade: "Os profissionais de nível superior da equipe possuem formação complementar que os qualifica para o trabalho na Atenção Básica" e "A equipe participa de cursos de atualização e qualificação, aplicando os conhecimentos aprimorados na melhoria do trabalho e da qualificação da atenção".

Cada item permite avaliar o grau de adequação das práticas da equipe de saúde da família ao padrão de qualidade apresentado, em uma escala que varia de $0 \mathrm{a}$ 10 pontos, e 0 refere-se ao não cumprimento ao padrão e 10 a total adequação. Os pontos somados classificam as equipes em padrões muito insatisfatório, insatisfatório, regular, satisfatório e muito satisfatório, conforme 0-3 pontos, 4-7, 8-11, 12-15 e 16-20, respectivamente (BRASIL, 2013). Quanto maior a pontuação melhor a classificação da equipe de saúde da família.

Além das questões do instrumento AMAQ, coletaram-se as seguintes informações das equipes de saúde da família: localização (urbana, rural); composição e número de profissionais na equipe - enfermagem, medicina, odontologia e agente comunitário de saúde.

A análise estatística foi realizada no Software IBM SPSS 22.0. Associaram-se as classificações das equipes em cada padrão de qualidade (muito insatisfatório a muito satisfatório) conforme localização (urbana, rural) pelo teste Likelihood ratio. As médias de pontos das equipes foram comparadas conforme localização (urbana e rural) pelo teste $t$ student. As médias de profissionais nas equipes foram comparadas conforme padrões de qualidade das equipes pelo teste Anova. A correlação entre os pontos obtidos pela equipe e o número de profissionais nas equipes foi executada pela correlação de Pearson. Todos os testes consideraram o nível de significância $p<0,05$.

$\mathrm{O}$ projeto de pesquisa deste estudo foi aprovado pelo Comitê de Ética em Pesquisa envolvendo Seres Humanos da Universidade Estadual de Montes
Claros, Unimontes - MG, parecer consubstanciado $\mathrm{n}^{\mathrm{o}}$ 704.718/2014 e foi conduzido em respeito aos princípios éticos contidos na resolução $\mathrm{n}^{0} 466 / 2012$, da Comissão Nacional de Ética em Pesquisa do Ministério da Saúde. A pesquisa contou com a concordância institucional da Secretaria Municipal de Saúde (SMS) do município estudado.

\section{RESULTADOS}

Observou-se que $18,7 \%$ das equipes pontuaram 10 para o item "Os profissionais da equipe de nível superior e médio possuem formação complementar que os qualifica para o trabalho na Atenção Básica" e, 25,3\% das equipes pontuaram 10 para o item "A equipe participa de cursos de atualização e qualificação, aplicando os conhecimentos aprimorados na melhoria do trabalho e da qualificação da atenção". Para os dois itens, mais de $80,0 \%$ das equipes pontuaram acima de cinco pontos (Tabela 1).

Tabela 1. Descrição da pontuação aos padrões de qualidade educação permanente e qualificação das equipes de Atenção Básica - MG, Brasil, 2014. n=75

\begin{tabular}{c|cc}
\hline $\begin{array}{c}\text { Escala de } \\
0 \text { a } 10\end{array}$ & \multicolumn{2}{|c}{$\begin{array}{c}\text { Educação permanente e qualificação das equipes } \\
\text { de Atenção Básica. Padrões de qualidade avaliados }\end{array}$} \\
\hline $\begin{array}{c}\text { Pontuação } \\
\text { das } \\
\text { equipes }\end{array}$ & $\begin{array}{c}\text { Profissionais } \\
\text { com formação } \\
\text { complementar para } \\
\text { Atenção Básica }\end{array}$ & $\begin{array}{c}\text { Participação em } \\
\text { cursos de atualização } \\
\text { e qualificação para } \\
\text { Atenção Básica }\end{array}$ \\
\cline { 2 - 3 } & $\mathrm{n}(\%)$ & $\mathrm{n}(\%)$ \\
\hline 0 & $1(1,3)$ & $0(0,0)$ \\
1 & $0(0,0)$ & $0(0,0)$ \\
2 & $0(0,0)$ & $0(0,0)$ \\
3 & $2(2,7)$ & $2(2,7)$ \\
4 & $1(1,3)$ & $3(4,0)$ \\
5 & $5(6,7)$ & $6(8,0)$ \\
6 & $8(10,7)$ & $10(13,3)$ \\
7 & $7(9,3)$ & $9(12,0)$ \\
8 & $17(22,7)$ & $19(25,3)$ \\
9 & $20(16,7)$ & $7(9,3)$ \\
10 & $14(18,7)$ & $19(25,3)$ \\
\hline
\end{tabular}


A pontuação máxima a ser alcançada por equipe seria de 20 pontos, ou seja, 10 em cada padrão de avaliação. Contudo, observou-se, neste estudo, uma variação de 8 a 20 pontos, com média igual a $15,2( \pm 3,417)$ pontos por equipe (Tabela 2).

Tabela 2. Valores médios e separatrizes dos pontos em educação permanente e qualificação das equipes de Atenção Básica - MG, Brasil, 2014. $\mathrm{n}=75$

\begin{tabular}{lc}
\hline Média & 15,2 \\
\hline Moda & 16,0 \\
\hline Desvio padrão & 3,417 \\
\hline Mínimo & 8,0 \\
\hline Máximo & 20,0 \\
\hline Percentil: & \\
\hline 25 & 14,0 \\
\hline 50 & 16,0 \\
\hline 75 & 18,0 \\
\hline
\end{tabular}

As classificações em padrões muito insatisfatório e insatisfatório não foram detectadas entre as equipes de saúde da família, e a maioria foi classificada como padrão de qualidade muito satisfatório (53,3\%), conforme Tabela 3.

Tabela 3. Classificação das equipes de Atenção Básica - MG, Brasil, 2014. $\mathrm{n}=75$

Educação permanente e qualificação das equipes de Atenção Básica

\begin{tabular}{lcc}
\hline $\begin{array}{l}\text { Classificação em } \\
\text { padrão }\end{array}$ & $\mathrm{n}$ & $\%$ \\
\hline $\begin{array}{l}\text { Muito } \\
\text { insatisfatório }\end{array}$ & 0 & 0,0 \\
$\begin{array}{l}\text { Insatisfatório } \\
\text { Regular }\end{array}$ & 0 & 0,0 \\
Satisfatório & 15 & 20,0 \\
$\begin{array}{l}\text { Muito } \\
\text { satisfatório }\end{array}$ & 20 & 26,7 \\
\hline Total & 40 & 53,3 \\
\hline
\end{tabular}

A correlação entre o número de profissionais e a pontuação pela equipe foi demonstrada na Tabela 4. A análise de correlação com profissionais da enfermagem não foi permitida porque todas as equipes de saúde apresentaram um profissional dessa categoria, ou seja, não houve variabilidade dos dados entre as equipes. A categoria profissional cirurgião-dentista correlacionouse positivamente, verificando-se que a presença de profissionais na equipe melhorou a pontuação na avaliação da equipe, e a variabilidade de uma variável em relação à outra foi explicada em 7,3\%. Contrariamente, o maior número de profissionais técnicos em enfermagem e de agentes comunitários de saúde representou menor pontuação da equipe, apesar de não significativa (Tabela 4).

Tabela 4. Correlação entre número de profissionais por categoria e pontos da autoavaliação das equipes de Atenção Básica - MG, Brasil, 2014. n=75

\begin{tabular}{|c|c|c|c|}
\hline \multirow{2}{*}{$\begin{array}{l}\begin{array}{l}\text { Número de profissionais por } \\
\text { equipe }\end{array} \\
\text { Categoria ( }{ }^{\circ} \text { mínimo e máximo } \\
\text { de profissionais) }\end{array}$} & \multicolumn{3}{|c|}{$\begin{array}{c}\text { Pontos na subdimensão } \\
\text { Educação Permanente e } \\
\text { qualificação das equipes de } \\
\text { Atenção Básica }\end{array}$} \\
\hline & $\mathrm{R}$ & $\mathrm{R}^{2}$ & $p$ \\
\hline Profissionais no geral $(8-11)$ & 0,145 & 0,021 & 0,215 \\
\hline Técnicos em enfermagem $(0-1)$ & $-0,040$ & 0,002 & 0,738 \\
\hline Médicos $(0-1)$ & 0,212 & 0,045 & 0,067 \\
\hline Cirurgiões-dentistas $(0-1)$ & 0,270 & 0,073 & 0,019 \\
\hline $\begin{array}{l}\text { Auxiliares/técnicos } \\
\text { odontologia }(0-2)\end{array}$ & 0,161 & 0,026 & 0,168 \\
\hline $\begin{array}{l}\text { Agentes comunitários de saúde } \\
(4-11)\end{array}$ & $-0,044$ & 0,002 & 0,710 \\
\hline
\end{tabular}

A média de profissionais em geral nas equipes classificadas como padrão de qualidade regular foi 11,20 $( \pm 1,781)$; para satisfatório, $10,60( \pm 1,353)$ e muito satisfatório, $11,48( \pm 1,062)$, sem diferença estatística entre as médias $(p=0,057)$ de profissionais conforme classificação das equipes.

A diferença entre as médias de pontos das equipes da zona rural e urbana foi estatisticamente significativa $(p=0,005)$, com maior pontuação para as equipes da zona urbana, $(15,66 \pm 3,252)$ que rurais, $(12,55 \pm 3,267)$.

A associação entre classificação das equipes conforme localização foi significativa, apenas 5,0\% das localizadas na zona rural foram classificadas como padrão muito satisfatório (Tabela 5). 
Tabela 5. Classificação das equipes de Atenção Básica conforme localização urbana ou rural - MG, Brasil, 2014. $n=75$

\begin{tabular}{l|cccc}
\multicolumn{5}{c}{ Educação permanente e qualificação das equipes de Atenção } \\
Básica
\end{tabular}

\section{DISCUSSÃO}

O presente estudo evidenciou predominância de autoavaliação positiva das equipes de saúde da família quanto à educação permanente e qualificação profissional para $\mathrm{AB}$, revelando que os profissionais se sentem satisfeitos, se preocupam com a formação para o trabalho e possuem formação qualificada para atuarem na $\mathrm{AB}$. Tal qualificação contribui para que se desenvolvam ações de saúde mais resolutivas no território e promotoras de melhorias nos processos de trabalho (BRASIL, 2013). Resultados similares foram verificados na literatura acerca da avaliação da $\mathrm{AB}$ e da educação permanente em saúde (LEITE et al., 2012; AURÉLIO PINTO et al., 2014; FONSECA SOBRINHO et al., 2014; SEIDL et al., 2014; YAMAMOTO; MACHADO; SILVA JUNIOR, 2015; TURCI; LIMA-COSTA; MACINKO, 2015; UCHÔA et al., 2016).

Os resultados observados nesta investigação podem ser explicados, provavelmente, pelo fato de que no município cenário deste estudo são ofertadas a Residência Multiprofissional em Saúde da Família e a Residência em Medicina de Família e Comunidade, além de especializações lato sensu em saúde da família e dos programas de pós-graduação stricto sensu em ciências da saúde e cuidados primários em saúde (GONÇALVES et al., 2014). O programa de Educação Permanente para Médicos de Família, também existente no município, constitui intervenção educacional da Secretaria de Estado da Saúde de Minas Gerais para promover a educação permanente dos médicos que atuam nas equipes de saúde da família. Baseia-se nos princípios da aprendizagem de adultos e nas melhores evidências científicas disponíveis na literatura internacional (LEITE et al., 2012).

Em adição, diante da relevância de qualificar e aperfeiçoar as práticas em saúde da família, e ampliar as possibilidades da formação de recursos humanos, instituições centram esforços na educação permanente de seus profissionais. Nos últimos anos, esse fortalecimento também tem sido potencializado pela oferta de cursos nas modalidades presencial e a distância, destinados principalmente aos profissionais que já atuam nos serviços de saúde (MATTOS; DAHMER; MAGALHÃES, 2015).

A literatura (LEITE et al., 2012; AURÉLIO PINTO et al., 2014; YAMAMOTO; MACHADO; SILVA JUNIOR, 2015; TURCI; LIMA-COSTA; MACINKO, 2015; UCHÔA et al., 2016) evidencia os benefícios da educação permanente para o processo de trabalho dos profissionais e a atenção à saúde da comunidade. Pesquisa realizada na região Norte de Minas Gerais demonstrou que as experiências de formação dos profissionais de saúde da família se aderem às concepções atuais de educação permanente, com potencial para transformação das práticas de saúde e organização das redes de atenção à saúde, embora se encontrem em processo de construção (LEITE et al., 2012). Estudo de Aurélio Pinto et al. (2014), elaborado com dados do PMAQ avaliou as equipes de Estratégia de Saúde da Família (ESF) do Brasil e revelou que a avaliação positiva das equipes é sugestiva de que há associação entre educação permanente em saúde e $\mathrm{AB}$, operadora do cotidiano do Sistema Único de Saúde (SUS) e do contato capilarizado com a população. Ademais, sinaliza-se a importância da continuidade e do incremento de ações no escopo da Política Nacional de Educação Permanente em Saúde.

Em Teresópolis - Rio de Janeiro, a prática de educação permanente foi capaz de promover mudanças no processo de trabalho, viabilizar a formação críticoreflexiva dos profissionais de saúde, fortalecer a participação social e aproximar a gestão das questões locais de saúde, comprovando que pode ser considerada uma prática avaliativa amistosa à integralidade (YAMAMOTO; MACHADO; SILVA JUNIOR, 2015). Em outra investigação que avaliou o desempenho da Atenção Primária à Saúde (APS) em Belo Horizonte - Minas Gerais, entre 
enfermeiros das equipes de saúde da família e gerentes, mostrou que a formação das equipes no tema saúde da família foi o segundo fator mais fortemente associado ao desempenho da APS, ressaltando a importância e a necessidade da formação específica na área (TURCI; LIMA-COSTA; MACINKO, 2015).

O desenvolvimento do curso de especialização em saúde da família apresenta potencial para provocar uma série de reflexões e alterações no cotidiano de prática dos trabalhadores, tais como a reorganização do processo de trabalho da equipe, a implementação de atividades coletivas, a implementação de acolhimento à demanda espontânea e maior compreensão do processo de trabalho de uma equipe da ESF (MATTOS; DAHMER; MAGALHÃES, 2015; UCHÔA et al., 2016). A relevância e a necessidade de educação permanente em saúde também são ratificadas ao se observar que, em países como Estados Unidos, Inglaterra e Alemanha, o uso de recursos denominados "telemedicina" (telemedicine, telebealth) tem ocorrido de forma sistemática e constante; especialmente mediante a organização de redes de serviços especializados para telediagnóstico e definição de medidas terapêuticas para casos específicos de patologias. São realizadas, ainda, algumas experiências com a utilização das tecnologias para o contato direto do médico com os usuários, em domicílio, visando à longitudinalidade dos cuidados domiciliares (AURÉLIO PINTO et al., 2014).

Todavia, diferentemente do constatado nesta pesquisa, em estudo de autoavaliação da qualidade da ESF na macrorregião Nordeste de Minas Gerais, a educação permanente esteve entre as subdimensões com pior avaliação (CARDOSO et al., 2015). No Estado do Paraná, as questões referentes à educação permanente evidenciaram fragilidades no âmbito das equipes que aderiram ao PMAQ, uma vez que 58,1\% dos trabalhadores afirmaram que as ações contemplaram as demandas e necessidades da equipe, ao passo que os demais responderam o inverso. Esses dados são contraditórios com o pressuposto da própria educação permanente em saúde, que deve ser constituída a partir das necessidades dos trabalhadores e, portanto, com a participação desses atores. Uma das possíveis explicações é que esses profissionais estejam participando de capacitações convencionais, definidas centralmente, e não se sentem protagonistas dos processos (RIZZOTTO et al., 2014).

Tal realidade verificada no Estado do Paraná também deve ser melhor investigada no presente trabalho, pois encontraram-se equipes com avaliação correspondente ao padrão de qualidade regular. Esse achado deve ser analisado com ponderação, pois indica que a educação permanente ainda se situa em um contexto de construção gradual.

Neste estudo, identificou-se que somente para a categoria profissional cirurgião-dentista houve correlação positiva e significativa com a pontuação da equipe, indicando que a presença desse profissional na equipe melhorou a pontuação na subdimensão investigada. Similarmente, em uma pesquisa descritiva multicêntrica, a partir de dados secundários da avaliação externa do PMAQ no Brasil, com 17.202 equipes de AB, quando comparadas às categorias profissionais, verificouse diferença significativa, com maior proporção tanto de médicos como de cirurgióes-dentistas que tenderam a referir aspectos mais positivos das suas unidades (UCHÔA et al., 2016).

Acredita-se que a presença de cirurgiões-dentistas na equipe pode contribuir para o compartilhamento de recursos e conhecimentos, além de melhoria na qualidade da APS e maior sustentabilidade de sua política (TURCI; LIMA-COSTA; MACINKO, 2015). Segundo Costa et al. (2014), a categoria profissional da área da odontologia, historicamente, se consolidou em quatro paredes, com assistência individual e ênfase no biologicismo e tecnicismo. Contudo, no âmbito da ESF, o resultado identificado no presente estudo pode sugerir superação do modelo tradicional de atenção à saúde e efetivação do novo paradigma de promoção de saúde nessa profissão.

Embora não tenha sido encontrado resultado, com diferença estatística significativa, o maior número de profissionais técnicos em enfermagem e de agentes comunitários de saúde representou pontuação inferior na subdimensão. Todavia, é pertinente refletir a respeito de tal achado, pois ainda na sociedade alguns indivíduos são pressionados a viver uma realidade alienada de cumprir tarefas e atividades, quantificando-as como produtividade e dissociadas do processo de gestão e criação (MATTOS; DAHMER; MAGALHÃES, 2015). 
Nesse sentido, vale salientar que a educação permanente em saúde não diz respeito somente a uma questão metodológica, na qual os problemas do serviço viriam à tona para resolvê-los. Para além desse pragmatismo, torna-se importante delinear os conteúdos que ampliem a visão dos trabalhadores, emitindo melhor compreensão do trabalho em saúde e de suas singularidades. Essa compreensão se torna possível se houver uma concepção dos trabalhadores como sujeitos de conhecimento e de cultura para, com isso, entender qual o sentido dos conhecimentos para esses sujeitos no exercício profissional em saúde. Nesse contexto, os desafios da formação de trabalhadores em saúde situam-se também em um plano epistemológico, especialmente quanto aos sentidos dos saberes formais ou conhecimentos científicos e dos saberes profissionais técnicos e sociopolíticos, expressos pela histórica e difícil relação entre teoria e prática (RAMOS, 2006; LEMOS, 2016).

No que se refere à localização das equipes de saúde da família, houve diferença significativa entre as situadas na zona urbana e rural, com classificação muito satisfatória apenas para 5,0\% das equipes localizadas na zona rural. Nos estudos sobre o PMAQ não foi possível identificar tal diferença (PINTO; SOUSA; FLORÊNCIO, 2012; FONSECA SOBRINHO et al., 2014; SEIDL et al., 2014; RIZZOTTO et al., 2014; AURÉLIO PINTO et al., 2014; UCHÔA et al., 2016). Possíveis explicações para tal achado podem envolver diversos aspectos. Mesmo diante das afirmações sobre o impacto e a ampliação da cobertura da ESF, persiste o desafio de superar as desigualdades em termos de acesso e qualidade da atenção, mesmo dentro dos municípios. É oportuno questionar se essa ampla cobertura representa uma contribuição à conversão de modelo assistencial ou uma reprodução na saúde da escassez e da desigualdade na distribuição de bens e equipamentos sociais, como observado nas demais esferas da vida social brasileira (UCHOA et al., 2011).

$\mathrm{E}$, apesar de vários investimentos para capacitar profissionais, os esforços precisam ser intensificados a fim de que as estratégias de educação permanente em saúde tragam benefícios em larga escala para a consolidação da ESF e de um sistema de saúde centrado na AB (ARANTES; SHIMIZU; MERCHÁN-HAMANN, 2016), desafio este certamente ainda mais complexo em áreas rurais.

Assim, para Leite et al. (2012), é pertinente ponderar que, no contexto da $\mathrm{AB}$, a educação permanente em saúde, ainda se encontra em processo de construção, como o processo de avaliação das equipes. As dificuldades envolvem questões de formação, gerenciais, institucionais e relativas aos processos de trabalho. Mediante o enfrentamento dessas dificuldades, podem surgir possibilidades de ação e caminhos estratégicos, que possibilitem mudanças nas práticas de atenção à saúde, segundo as necessidades reais dos serviços, das famílias e da comunidade.

Por fim, os resultados da presente pesquisa devem ser considerados à luz de certas limitações. Tratase de um estudo com delineamento transversal, sem possibilidade de definir uma relação de causalidade para as associações identificadas. Foi circunscrito ao cenário das unidades da ESF de um município. E não foram utilizadas técnicas de observação direta, mas, sim, analisados dados oriundos da autoavaliação das equipes de saúde da família, que podem estar propensos a um possível viés de informação.

\section{CONCLUSÃO}

O estudo evidenciou predominância de avaliação positiva das equipes quanto à educação permanente e qualificação profissional para o cuidado na $\mathrm{AB}$. No entanto, constatam-se equipes classificadas no padrão de qualidade regular, sugerindo que a educação permanente e qualificação profissional ainda não se configuraram como ferramentas no cotidiano das equipes, em especial para os profissionais que atuam na zona rural. Observouse, também, que a presença do profissional cirurgiãodentista na equipe contribuiu para uma avaliação mais positiva.

Recomenda-se que a educação permanente e a autoavaliação integrem a realidade do cotidiano de trabalho das equipes e as necessidades reais dos serviços públicos de saúde. É preciso planejar ações de incentivo à qualificação profissional, em serviço e continuamente pela educação permanente, para aquisição de competências e habilidades necessárias ao dinamismo do cuidado na $\mathrm{AB}$. 


\section{REFERÊNCIAS}

ARANTES, L. J.; SHIMIZU, H. E.; MERCHÁN-HAMANN, E. The benefits and challenges of the Family Health Strategy in Brazilian Primary Health care: a literature review. Ciênc Saúde Coletiva, Rio de Janeiro, v. 21, n. 5, p. 1499-1510, 2016.

AURÉLIO PINTO, H.; FERLA, A. A.; CECCIM, R. B.; FLORÊNCIO, A. R.; MATOS, I. B.; BARBOSA, M. G.; STÉDILE, N. L. R.; ZORTEA, A. P. Atenção Básica e Educação Permanente em Saúde: cenário apontado pelo Programa Nacional de Melhoria do Acesso e da Qualidade da Atenção Básica (PMAQ-AB). Divulg Saúde Debate, Rio de Janeiro, v. 51, p. 145-160, 2014.

BRASIL. Instituto Brasileiro de Geografia e Estatística IBGE, Cidades@. Minas Gerais: Montes Claros. Rio de Janeiro (RJ): Instituto Brasileiro de Geografia e Estatística, 2015. Disponível em: < http://cidades.ibge.gov.br/xtras/ perfil.php?codmun=314330 > . Acesso em: 22 ago 2016.

BRASIL. Ministério da Saúde. Secretaria de Atenção à Saúde. Departamento de Atenção Básica. AMAQ: autoavaliação para melhoria do acesso e da qualidade. Brasília (DF): Ministério da Saúde, 2013.

BRASIL. Portaria n. 198/GM, de 13 de fevereiro de 2004. Institui a Política Nacional de Educação Permanente em Saúde como estratégia do Sistema Único de Saúde para a formação e o desenvolvimento de trabalhadores para 0 setor e dá outras providências. Brasília (DF): Ministério da Saúde, 2004.

CARDOSO, A. V. L.; CHAIN, A. P. N.; MENDES, R. I. P.; FERREIRA E FERREIRA, E.; VARGAS, A. M. D.; MARTINS, A. M. E. B. L.; FERREIRA, R. C. Assessment of the management of the Family Health Strategy via the tool Assessment for Quality Improvement in municipalities of Minas Gerais, Brazil. Ciênc Saúde Coletiva, Rio de Janeiro, v. 20, n. 4, p. 1267-1284, 2015.

CECCIM, R. B. Educação Permanente em Saúde: desafio ambicioso e necessário. Interface, Botucatu, v. 9, n. 16, p. 161-168, 2005.

COSTA, S. M.; SOUZA E SOUZA, L. P.; SOUZA, T. R.; CERQUEIRA, A. L. N.; BOTELHO, B. L.; ARAÚJO, E. P.
P.; RODRIGUES, C. A. Q. Práticas de trabalho no âmbito coletivo: profissionais da equipe Saúde da Família. Cad Saúde Colet, Rio de Janeiro, v. 22, n. 3, p. 292-299, 2014.

FONSECA SOBRINHO, D.; MACHADO, A. T. G. M.; LIMA, A. M. L. D.; OLIVEIRA, J. A.; REIS, C. M. R.; ABREU, D. M. X.; ARAÚJO, L. H. L.; EVANGELISTA, M. L. F.; ESCOBAR, A. L.; SANTOS, C. R. I.; SANTOS, A. F. Compreendendo o apoio matricial e o resultado da certificação de qualidade nas áreas de atenção à criança, mulher, diabetes/hipertensão e saúde mental. Saúde Debate, Rio de Janeiro, v. 38, n. especial, p. 83-93, 2014.

GONÇALVES, C. R.; CRUZ, M. T.; OLIVEIRA, M. P.; MORAIS, A. J. D.; MOREIRA, K. S.; RODRIGUES, C. A. Q.; LEITE, M. T. S. Recursos humanos: fator crítico para as redes de atenção à saúde. Saúde Debate, Rio de Janeiro, v. 38, n. 100, p. 26-34, 2014.

LEITE, M. T. S.; SENA, R. R.; VIEIRA, M. A.; MENDONÇA, J. M. G.; DIAS, O. V.; SANTOS, M. I. P.; SOUZA E SOUZA, L. P. Perspectivas de educação permanente em saúde no Norte de Minas Gerais. REME - Rev Min Enferm, Belo Horizonte, v. 16, n. 4, p. 594-600, 2012.

LEMOS, C. L. S. Educação Permanente em Saúde no Brasil: educação ou gerenciamento permanente? Ciênc Saúde Coletiva, Rio de Janeiro, v. 21, n. 3, p. 913-922, 2016.

MATTOS, L. B.; DAHMER, A.; MAGALHÃES, C. R. Contribuição do curso de especialização em Atenção Primária à Saúde à prática de profissionais da saúde. ABCS Health Sci, v. 40, n. 3, p. 184-189, 2015.

PINTO, H. A.; SOUSA, A.; FLORÊNCIO, A. R. The National Program for Improving the Access and Quality of Primary Care: reflections on the program design and deployment process. Rev Eletron Comun Inf Inov Saúde, v. 6, n. 2, 2012. Disponível em: < http://www.reciis.icict.fiocruz. br/index.php/reciis/article/view/624/1083:. Acesso em: 14 jan 2016.

PORTELA, L. R.; DIAS, M. S. A.; VASCONCELOS, M. I. O. Programa Nacional da Melhoria do Acesso e da Qualidade da Atenção Básica: análise da Autoavaliação em Sobral, Ceará. Sanare, Sobral, v. 12, n. 1, p. 40-45, 2013. 
RAMOS, M. N. Referências teórico-metodológicas da educação permanente em saúde no Brasil. Rio de Janeiro: Fiocruz, 2006.

RIZZOTTO, M. L. F.; GIL, C. R. R.; CARVALHO, M.; FONSECA, A. L. N.; SANTOS, M. F. Força de trabalho e gestão do trabalho em saúde: revelações da Avaliação Externa do Programa Nacional de Melhoria do Acesso e da Qualidade da Atenção Básica no Paraná. Saúde Debate, Rio de Janeiro, v. 38, n. especial, p. 237-251, 2014.

SEIDL, H.; VIEIRA, S. P.; FAUSTO, M. C. R.; LIMA, R. C. D.; GAGNO, J. Gestão do trabalho na Atenção Básica em Saúde: uma análise a partir da perspectiva das equipes participantes do PMAQ-AB. Saúde Debate, Rio de Janeiro, v. 38, n. especial, p. 94-108, 2014.

TURCI, M. A.; LIMA-COSTA, M. F.; MACINKO, J. Influência de fatores estruturais e organizacionais no desempenho da atenção primária à saúde em Belo Horizonte, Minas Gerais, Brasil, na avaliação de gestores e enfermeiros. Cad Saúde Pública, Rio de Janeiro, v. 31, n. 9, p. 19411952, 2015.

UCHOA, A. C.; SOUZA, E. L.; SPINELLI, A. F. S.; MEDEIROS, R. G.; PEIXOTO, D. C. S.; SILVA, R. A. R., ROCHA, N. S. P. Avaliação da satisfação do usuário do Programa de Saúde da Família na zona rural de dois pequenos municípios do Rio Grande do Norte. Physis, Rio de Janeiro, v. 21, n. 3, p. 1061-1076, 2011.

UCHÔA, S. A. C.; ARCÊNCIO, R. A.; FRONTEIRA, I. S. E.; COÊLHO, A. A.; MARTINIANO, C. S.; BRANDÃO, I. C. A.; YAMAMURA, M.; MAROTO, R. M.; SILVA, A. K. F. Potential access to primary health care: what does the National Program for Access and Quality Improvement data show? Rev Latino-Am Enfermagem, Ribeirão Preto, v. 24, p. e2672, 2016.

YAMAMOTO, T. S.; MACHADO, M. T. C.; SILVA JUNIOR, A. G. Educação permanente em saúde como prática avaliativa amistosa à integralidade em Teresópolis, Rio de Janeiro. Trab Educ Saúde, Rio de Janeiro, v. 13, n. 3, p. 617-637, 2015.
Recebido em: 02 de agosto de 2016 Aceito em: 15 de abril de 2017 